\title{
USING GPS MULTIPATH FOR SNOW DEPTH SENSING - FIRST EXPERIENCE WITH DATA FROM PERMANENT STATIONS IN SLOVAKIA
}

\author{
Ján HEFTY * and L’ubomíra GERHÁTOVÁ \\ Department of Theoretical Geodesy, Faculty of Civil Engineering Slovak University of Technology, \\ Radlinského 11, 81368 Bratislava, Slovak Republic \\ *Corresponding author's e-mail: jan.hefty@stuba.sk
}

\begin{tabular}{l} 
ARTICLE INFO \\
\hline Article history: \\
Received 29 July 2013 \\
Accepted 17 September 2013 \\
Available online 26 November 2013 \\
\hline
\end{tabular}

Keywords:

GPS multipath

Satellite signals reflected from ground Signal-to-noise ratio

Geometry-free linear combination of carrier phases

\begin{abstract}
Analysis of multipath reflections of GPS satellite signals at permanent GPS stations allows deducing the height of GPS antenna above the horizontal reflecting surface. If the effective height of the reflector is varying, e.g. due to change of snow cover in the vicinity of GPS antenna monumentation, the multipath characteristics are affected, namely the period of the harmonic oscillations of the reflected signal. We analyzed three types of GPS observables influenced by multipath: signal-to-noise ratio SNR1 and SNR2 related to L1 and L2 carrier phases respectively, and L4, the geometry-free linear combination of carrier phases. The GPS multipath snow sensing is demonstrated on two GNSS permanent stations in Slovakia - Gánovce (GANP) and Liesek (LIE1), where the snow cover during winter season reaches up to $40 \mathrm{~cm}$ and the snow depth manual measurements are regularly performed. Due to terrain topography, buildings, structures and other obstacles in the vicinity of pillar where the GPS antenna is mounted only narrow azimuthal sectors in these sites are suitable for analyzing reflected signals from ground. Comparisons of series of manual snow depth sensing with data resulting from GPS multipath analyses generally shows consistency better than $5 \mathrm{~cm}$, however some periods where the biases are at $10 \mathrm{~cm}$ level were recognized.
\end{abstract}

\section{INTRODUCTION}

Multipath in GPS observations is generally considered as a disturbing factor systematically influencing static and kinematic positioning. The broadcasted satellite microwave signal directly reaching the GPS antenna is interfered with the satellite signal reflected from the buildings and terrain around the antenna - systematical effect which is usually described in publications dealing with precise geodetic satellite surveying e.g. in (HoffmannWellenhof et al., 2001). Motion of the GPS satellite on the sky as well as the features of the reflection surface affects the multipath characteristics, namely its spatial and temporal variability. Recently, several studies have shown that the analysis of multipath caused by reflections from horizontal surface at permanently observing GPS sites allows to infer the properties of the environment of the antenna, namely the variability of depth of the reflecting surface and consecutively to deduce the variations of soil moisture or the changes of snow cover. Two types of GPS observables are efficient for such analyses of multipath: the signal-to-noise ratio (SNR) used by Larson (Larson et al., 2008, Larson et al., 2009) or the geometry-free linear combination of carrier phases (L4) applied by (Ozeki and Heki, 2011). The pseudorange/carrier phase multipath combinations MP1 and MP2 (Estey and Meertens, 1999) conventionally applied for multipath assessment are not suitable for monitoring the multipath time variability as long as the centimeter accuracy of the height of reflecting surface is expected.

We examined and compared both approaches on several permanent GPS stations in Slovakia, situated in various environments. The selection of GPS sites suitable for testing the potential of snow depth sensing was limited by various factors, namely: GPS antenna monumentation on stable pillar surrounded by flat ground, no vertical obstacles around the pillar (at lest in some sectors), continuous GPS observations in the winter period, snow cover reaching several tens of centimeters, availability of SNR data, field meteo observations of local temperature and manual measurements of snow depth. In this paper we will present results from data analyses related to two permanent stations: the IGS and EPN station Gánovce (GANP) and the permanent station Liesek (LIE1) belonging to the Slovak Positioning Service SKPOS. Both stations have GNSS antenna mounted on $\approx 3 \mathrm{~m}$ high concrete pillar and the preliminary analysis of the consistency of SNR and L4 data proved that the sites are suitable for analysis of multipath variability related to snow depth sensing at least in some azimuth sectors.

\section{APPLICATION OF GPS MULTIPATH VARIABILITY MONITORING FOR SNOW DEPTH SENSING}

The simplified geometrical model of multipath considering the reflections from horizontal planar 

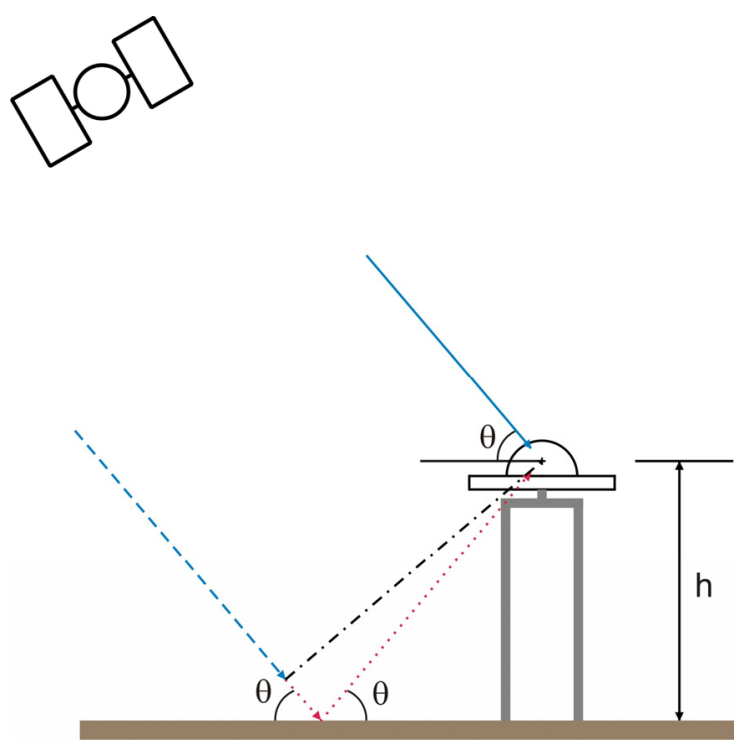

Fig. 1 Geometrical model of multipath considering the reflections from horizontal planar surface. Path of the direct satellite signal reaching the GPS antenna is prolonged by the additional path represented by dotted line. For elevation angle $\theta$ and antenna phase center height $h$ above the terrain the additional path is $2 h \sin \theta$.

surface around the GPS antenna is shown in Figure 1. The path of the direct satellite signal reaching the GPS antenna is prolonged by the additional path represented by dotted line. For satellite elevation angle $\theta$ the additional path is proportional to the antenna phase center height $h$ above the terrain for the value $2 h \sin \theta$ (Hoffmann-Wellenhof et al., 2001, p. 128). The multipath phase difference $\psi$ between the direct and indirect signal for the GPS carrier wavelength $\lambda$ is then

$\psi=\frac{4 \pi h}{\lambda} \sin \theta$

During the satellite continuous tracking the elevation angle $\theta$ is slowly changing (rising or setting of the satellite is function of time $t$ ) which causes the time variability of $\psi$. Then the frequency $\omega_{t}$ of the multipath variability can be expressed as timederivative of $\psi$

$\omega_{t}=\frac{d \psi}{d t}=\frac{4 \pi h}{\lambda} \cos \theta \frac{d \theta}{d t}$

As proposed by Larson et al. (2008), this equation can be modified and simplified by using the independent variable $s=\sin \theta$ instead of $t$ and then the frequency $\omega_{s}$ of multipath variability is expressed as

$\omega_{s}=\frac{d \psi}{d s}=\frac{4 \pi h}{\lambda}$
Interference of the direct satellite signal with amplitude $A_{d}$ and the reflected indirect signal with amplitude $A_{r}$ cause the change of signal strength $S$ and multipath induced carrier wave phase shift $\delta \varphi$ of the complete received signal (direct and indirect). According to (Billich et al., 2008; Ozeki and Heki, 2011) they are expressed as functions of multipath parameters $\psi, A_{d}$ and $A_{r}$ (or $\alpha=A_{r} / A_{d}$ ) as

$S^{2}=A_{d}^{2}+A_{r}^{2}+2 A_{d} A_{r} \cos \psi$

$\tan (\delta \phi)=\frac{A_{r} \sin \psi}{A_{d}+A_{r} \cos \psi}=\frac{\alpha \sin \psi}{1+\alpha \cos \psi}$

The time variability of $\psi$ due to slow change of elevation angle $\theta$ is manifested as periodic oscillations of signal strength $S$ and phase shift $\delta \varphi$. As coefficient of the microwave reflectivity at the ground is small ( $\alpha \approx 0.15$ ) the periodic part $S_{P}$ of signal strength can be approximated as

$S_{P}\left(A_{d}, \alpha, h, \lambda, \theta\right) \approx \alpha A_{d} \cos \left(\frac{4 \pi h}{\lambda} \sin \theta\right)$

and the phase shift is

$\delta \phi(\alpha, h, \lambda, \theta)=\tan ^{-1}\left[\frac{\alpha \sin \left(\frac{4 \pi h \sin \theta}{\lambda}\right)}{1+\alpha \cos \left(\frac{4 \pi h \sin \theta}{\lambda}\right)}\right]$

Analyses of series of satellite signal strength and carrier phase as functions of elevation angle $\theta$ enable to estimate the value of actual antenna height $h$ for the GPS signal with wavelength $\lambda$. The period of $S$ and $\delta \varphi$ decreases with increasing $h$ according to (3), (6) and (7).

The possibility of obtaining the actual height $h$ of antenna phase centre above the surrounding reflecting surface from multipath analysis enables to detect the potential variability of the height of the reflector provided the stable monumentation of GPS antenna. Application for snow depth sensing is demonstrated in Figure 2, where $h_{G}$ is the antenna height above ground without snow cover. Then the $h_{S N O W}$ is simply deduced using actual $h$ estimated from multipath as

$h_{\text {SNOW }}=h_{G}-h$

According to (6) and (7) two types of GPS observables are suitable for estimation of $h$, namely signal-to-noise ratio (SNR) and carrier phase, both generally included in GPS RINEX observation files (Gurtner, 1994).

The SNR data used for multipath studies e.g. in (Larson et al., 2009; Larson and Nievinski, 2012; Billich et al., 2008) have the advantage in their smoothness and independence of ionosphere fluctuations. The drawback of SNR observables is that they are reported in logarithmic scale (in $\mathrm{dB}$ ), their 

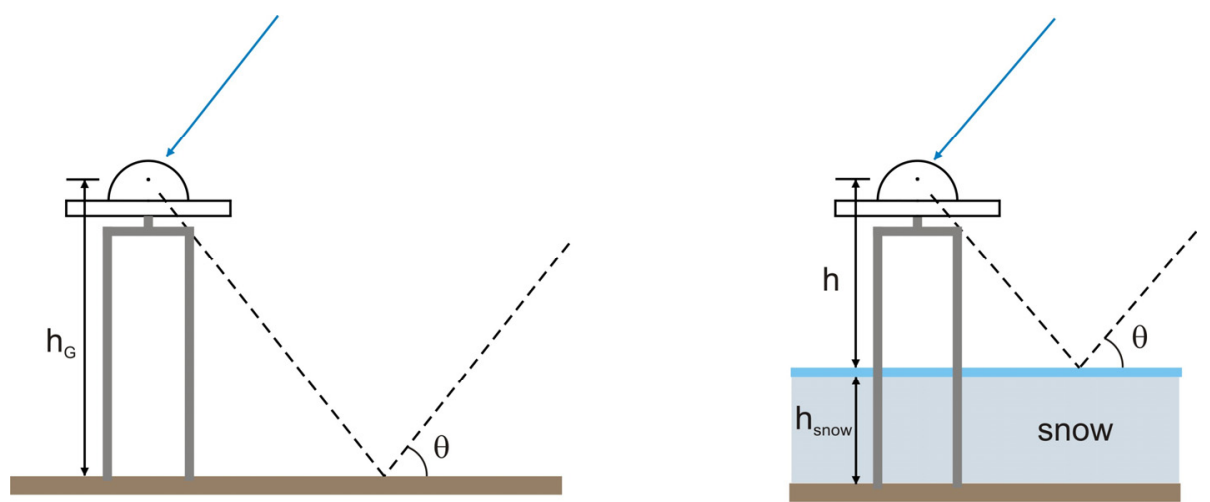

Fig. 2 Effect of snow coverage with $h_{S N O W}$ on the effective antenna height $h$ above the reflecting surface. If the value $h_{G}$ represents the effective antenna height above ground without snow coverage then $h_{S N O W}=h_{G}-h$.

resolution is in some cases limited as they are rounded to $1 \mathrm{~dB}$, and the value of SNR is receiver depended, according to the applied code correlation technique (Hoffmenn-Wellenhof et al., 2001). Moreover the SNR is not always included in all RINEX data files which are archived in GNSS data centres.

The geometry-free linear combination L4 of carrier phases used by (Ozeki and Heki, 2011) is simple difference between phase observations L1 L2, where the carrier phases are multiplied by wavelengths $\lambda_{1} \approx 19.0 \mathrm{~cm}$ and $\lambda_{2} \approx 24.4 \mathrm{~cm}$. The geometrical content of L4 is removed by differentiation and reduction for initial ambiguities. The time variability of L4 is then dependent on ionosphere disturbances and multipath issues only. The L1 and L2 phase observables are always included RINEX files, non-rounded and with sufficient temporal resolution.

\section{STRATEGY FOR ESTIMATION OF ACTUAL ANTENNA HEIGHT FROM SIGNAL-TO-NOISE RATIO AND GEOMETRY-FREE LINEAR COMBINATION OF CARRIER PHASES}

Multipath periodic fluctuations presented in signal-to-noise ratio series as well as in series of L4 linear combination of carrier phases can be applied for estimate of the effective antenna phase centre height $h$ above the reflecting horizontal surface. If the observation RINEX files include carrier phase data L1 and L2, as well as SNR data for L1 and L2 frequencies (denoted here as SNR1 and SNR2) it will be possible to estimate $h$ independently from three independent observables.

The GPS observations are recorded and stored with sampling rate $15 \mathrm{~s}$ or $30 \mathrm{~s}$. In order to apply eq. (3) for evaluation of effective antenna height $h$ from frequency $\omega_{s}$ of multipath induced periodic variations the original time argument $t$ of the series SNR1, SNR2 and L4 in RINEX files is transformed to new argument $s$ - the independent variable $s=\sin \theta$ for each analyzed satellite. The actual elevation angle $\theta$ of the observed satellite at epoch $t$ is computed from satellite ephemeris and site coordinates.
The SNR series during the satellite observations are sum of the direct signal component which generally increases with satellite elevation and the multipath component including the ground reflections. A multipath effect is diminishing with increase of satellite elevation angle and for study of multipath effect is sufficient to analyse the SNR below $30^{\circ}$ elevation. The SNR values given in RINEX files expressing the ratio of signal power to power of noise are in logarithmic scale in dB (Gurtner, 1994, Misra and Enge, 2001). For further analyses related to study of the multipath periodic variability the $S N R_{d B}$ data are converted to the ratio of signal amplitude to noise amplitude expressed in linear scale $S N R_{\text {lin }}$ according to the formula $S N R_{\text {lin }}=10^{S N R_{d B} / 20}$ (Billich et al., 2008).

The long-term part of the $S N R_{\text {lin }}$ due to satellite elevation change is removed by low-order $(n=5)$ polynomial. The frequency of periodic part is estimated from the residual series $\delta S N R$ by means of spectral analysis. The residual $\delta S N R 1$ and $\delta S N R 2$ series from SNR1 and SNR2 (transformed to linear scale as $S N R 1_{\text {lin }}$ and $S N R 2_{\text {lin }}$ ) are formed and analysed separately. If the reflecting surface is horizontal, smooth and no other obstacles are contributing to multipath pattern only one peak should be dominant in the spectra of each series.

Variations of L4 series besides the multipath from ground reflections are strongly influenced also by the time varying ionosphere delays. Similarly to SNR, only the observations below $30^{\circ}$ elevation angle are analysed. The disturbing unpredictable ionosphere influences are removed by polynomial of higher order $(n=14)$ and the residual series $\delta L 4$ is used for estimation of dominant frequencies corresponding to multipath from reflections from horizontal surface. Unlike to $\delta S N R 1$ or $\delta S N R 2$ in $\delta L 4$ two dominant peaks in spectra are expected, as the L4 is composed from two carriers L1 and L2, each separately influencing the multipath pattern of the geometry-free linear combination. 

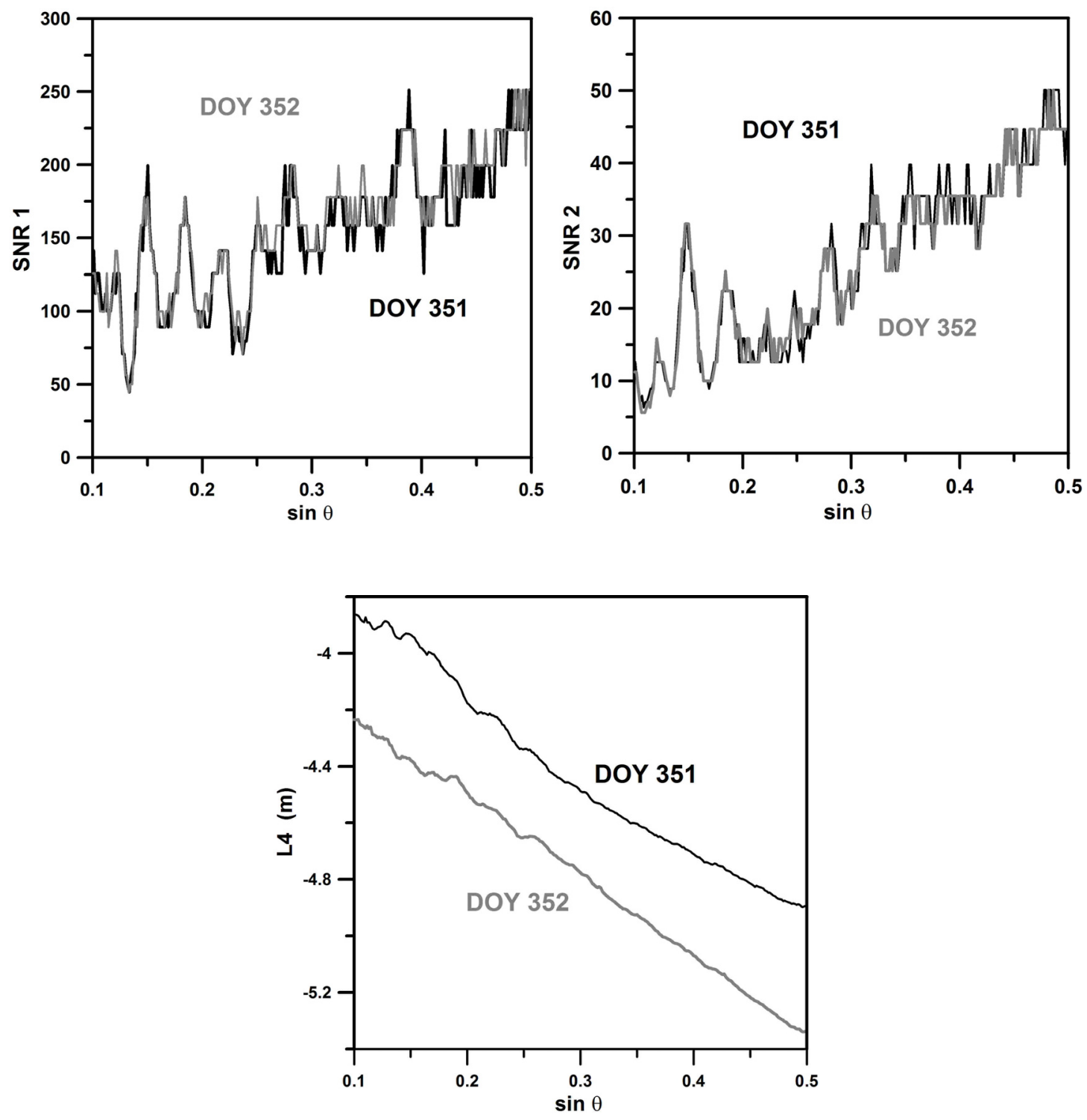

Fig. 3 Observed series of SNR1, SNR2 (transformed to linear scale) and and L4 for rising satellite PRN23 (elevation angle $\theta$ from $5^{\circ}$ to $30^{\circ}$ ) for two successive days. The original time argument of all the series was transformed to argument $s=\sin \theta$.

The purpose of spectral analyses of the observed $\delta S N R 1, \delta S N R 2$ and $\delta L 4$ series is to obtain multipath frequencies $\omega_{s}=d \psi / d s$. In the analyzed series the regular sampling time interval $t$ was transformed to the irregularly spaced sampling interval $s=\sin \theta$. In such case the spectral analysis method handling unequally spaced data has to be applied. We used here the least-squares spectral analysis approach (Wells et al., 1985) which besides the dominant frequencies estimates also their amplitudes and level of noise. The maximum spectral peak of $\delta S N R 1$ series corresponds to frequency $\omega_{S 1}$, the maximum spectral peak of $\delta S N R 2$ series to $\omega_{S 2}$, and two local maxima in the spectrum of $\delta L 4$ series correspond to frequencies $\omega_{L 1}$ and $\omega_{L 2}$.

Then the from equation (3) four estimates of effective antenna height $h$ are obtained as
$h_{S 1}=\frac{\omega_{S 1} \lambda_{1}}{4 \pi}, \quad h_{S 2}=\frac{\omega_{S 2} \lambda_{2}}{4 \pi}$,

$h_{L 1}=\frac{\omega_{L 1} \lambda_{1}}{4 \pi}, \quad h_{L 2}=\frac{\omega_{L 2} \lambda_{2}}{4 \pi}$

Examples of observation series of SNR1, SNR2 (transformed to linear scale) and L4 for rising satellite (elevation angle $\theta$ from $5^{\circ}$ to $30^{\circ}$ ) for two successive days are shown in Figure 3. The original argument $t$ of all the series was transformed to $s=\sin \theta$. The residual series $\delta S N R 1, \delta S N R 2$ and $\delta L 4$ which are obtained after elimination if long-term variation modeled by polynomial $\left(5^{\text {th }}\right.$ order for SNR1, SNR2 and $14^{\text {th }}$ order for L4) are shown in Figure 4. Such residual series are later on submitted to leastsquarer spectral analysis to find the dominant frequencies relating to the multipath due to reflections 

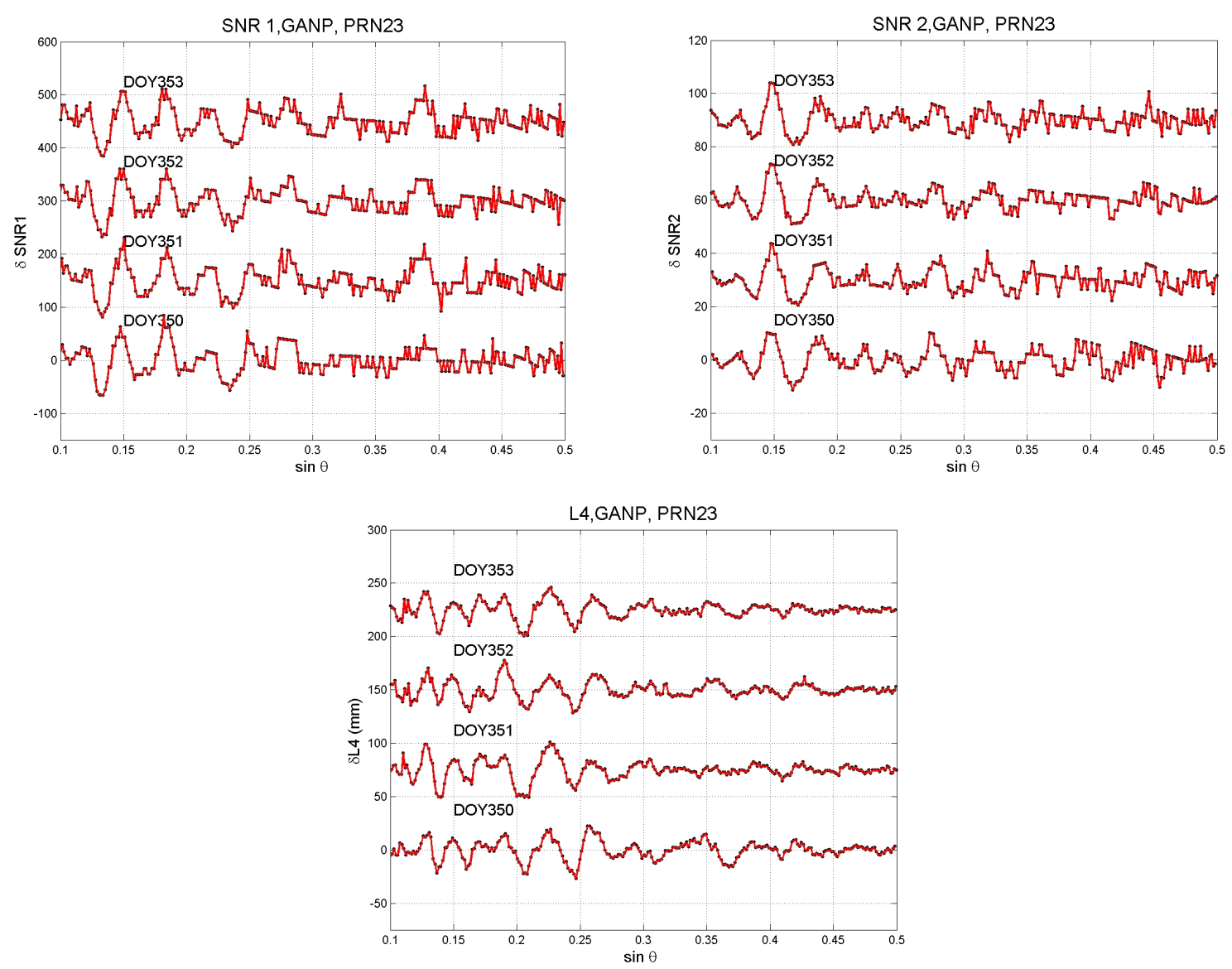

Fig. 4 Observed series of SNR1, SNR2 (transformed to linear scale) and L4 after reduction for low-frequency variations. Reduced series denoted as $\delta S N R 1, \delta S N R 2$ and $\delta L 4$ represent the repeating pattern of multipath effect for four successive days.

from the horizontal surface around the GPS antenna. Figure 5 shows example from permanent station GANP for two days: DOY 344 of 2011 without snow cover and for DOY 43 of 2012 with snow depth $25 \mathrm{~cm}$. On the horizontal axes of the amplitude spectra are periods $P$ of argument $\sin \theta$ which are related to frequencies $\omega$ used in (9) as $\omega=1 / P$. The comparison of dominant periods for each of the data series documents the shift of the spectra peak related to change of horizontal reflector height.

\section{RESULTS FROM GPS SNOW DEPTH SENSING AT STATION GANP (WINTER 2011/2012) AND LIE1 (WINTER 2012/2013)}

The applicability of multipath analyses for snow depth sensing at Slovak permanent GNSS stations will be demonstrated on IGS and EPN permanent station Gánovce (GANP) situated close to town Poprad and permanent GNSS station Liesek (LIE1) which is a part of the Slovak Positioning Service SKPOS situated in North Slovakia, close to the Orava Reservoir. Both stations have GNSS antenna mounted on $\sim 2.5 \mathrm{~m}$ concrete pillar situated in slightly undulating terrain. The basic information about the station location and station equipment are summarized in Table 1.

In the winter season the snow cover in GANP and LIE1 reaches up to $40 \mathrm{~cm}$. As it is visible from Figures 6 and 7, there are several buildings and other constructions surrounding the pillars, however free sectors suitable for analysis of multipath reflections from ground are available. The reflection areas for some satellites which we found usable for snow depth sensing are demonstrated by First Fresnel Zones (FFZ) in Figures 6 and 7. They were computed for the terrain without snow according to formulae given in (Larson and Nievinski, 2012) for satellites observed at elevation angles $\theta=7^{\circ}, 15^{\circ}$ and $25^{\circ}$ on carrier wave L2 with wavelength $\lambda_{2}$. Reflections for satellites observed at low elevation angles cover the area up to $50 \mathrm{~m}$ from antenna pillar. Snow cover decreases the effective height $h$ and diminishes the reflecting area. The $0.5 \mathrm{~m}$ snow layer reduces about $15 \%$ the maximum distance from pillar applied for estimation of dominant multipath frequencies.

The fundamental requirement for successful estimation of effective antenna height $h$ from (9) is identification of clear spectral peak corresponding to 

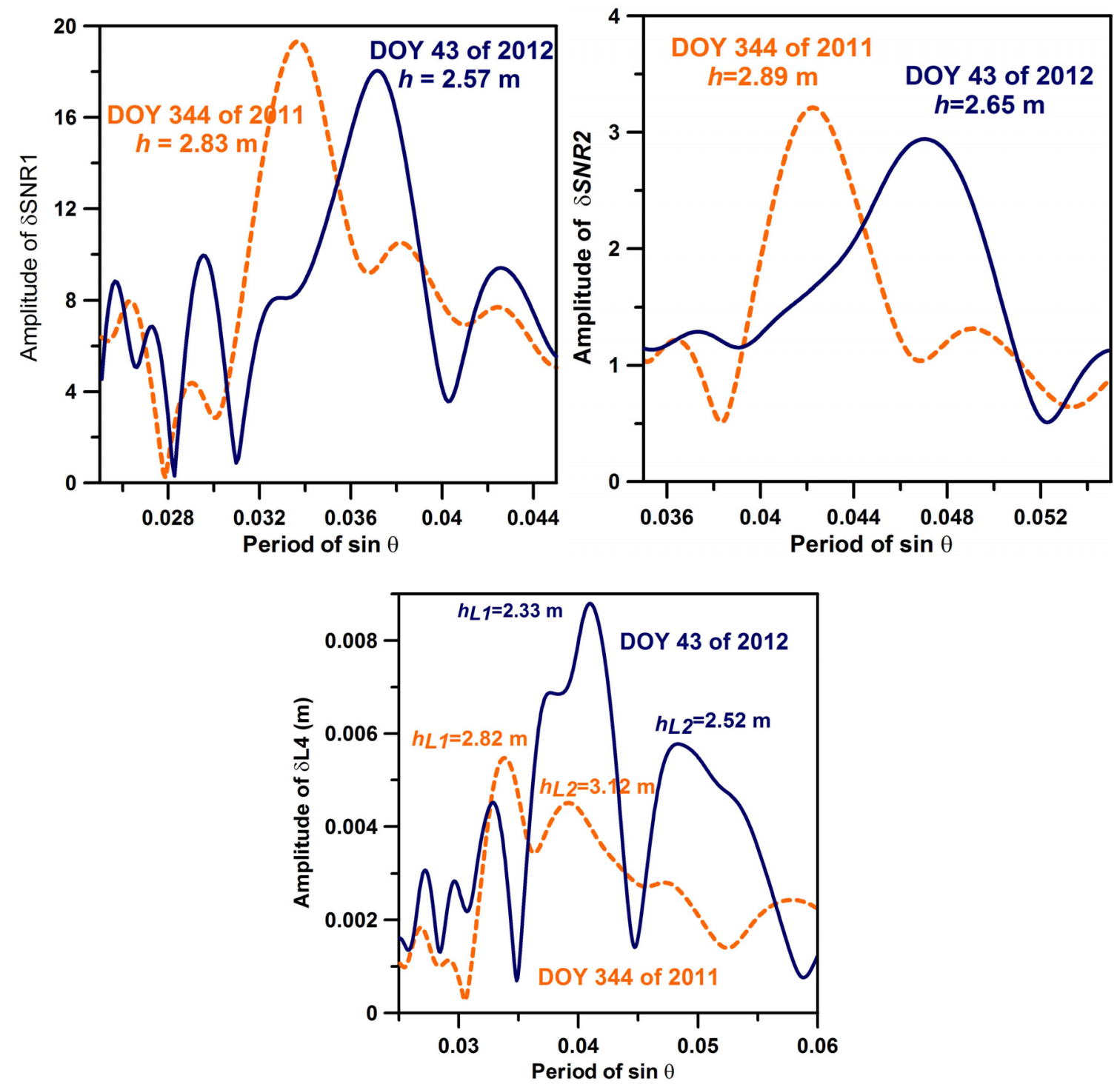

Fig. 5 Examples of amplitude spectra of $\delta S N R 1, \delta S N R 2$ and $\delta L 4$ from permanent station GANP for two days: DOY 344 of 2011 without snow cover and for DOY 43 of 2012 with snow depth $\sim 25 \mathrm{~cm}$. Comparison of dominant periods for each of the data series documents the shift of the spectra peak related to change of horizontal reflector height.

Table 1 Analyzed stations

\begin{tabular}{cccc}
\hline $\begin{array}{c}\text { GNSS permanent } \\
\text { station }\end{array}$ & $\begin{array}{c}\text { Latitude } \\
\text { Longitude } \\
\text { Elevation }\end{array}$ & $\begin{array}{c}\text { Receiver } \\
\text { Antenna }\end{array}$ & $\begin{array}{c}\text { Antenna height } \\
\text { (pillar } \\
\text { + centering rod } \\
\text { phase center) }\end{array}$ \\
\hline Gánovce & $49^{\circ} 02^{\prime} 05^{\prime \prime}$ & TRIMBLE NETR8 & $2.76 \mathrm{~m}$ \\
GANP & $20^{\circ} 19^{\prime} 23^{\prime \prime}$ & TRM55971.NONE & \\
& $704 \mathrm{~m}$ & & $3.10 \mathrm{~m}$ \\
Liesek & $49^{\circ} 22^{\prime} 09^{\prime \prime}$ & TRIMBLE NETR9 & \\
LIE1 & $69^{\circ} 40^{\prime} 42^{\prime \prime}$ & TRM59800.NONE & \\
& $692 \mathrm{~m}$ & & \\
\hline
\end{tabular}


dominant frequency $\omega$ in the $\delta S N R$ series and two dominant spectral peaks in the $\delta L 4$ series. For snow depth sensing is essential that the clearly distinguished spectral maxima are available for the processed rising or descending satellite during the whole winter season. If the GPS antenna pillar is situated in environment with various obstacles and variable terrain topography the choice of relevant satellites continuously observable above horizon up to $30^{\circ}$ is limited. The restrictions mentioned significantly reduce the number of potential satellites and sectors suitable for continuous snow sensing during the winter season. We found for GANP and LIE1 sites only several (3 - 4) azimuthal sectors with rising or descending satellites which were convenient for consistent analyses of multipath spectra and estimation of time changes of effective antenna height related to snow depth variability. In this paper we present the results obtained from descending satellite PRN 23 at site GANP (Fig. 6) and from the rising satellite PRN15 at site LIE1 (Fig. 7). It is obvious that such results will be representative only for narrow azimuth sector. The reliability of the estimated effective antenna height will be based on combination of separate estimates $h_{S 1}, h_{S 2}$ and $h_{L 4}$ from $\delta S N R 1$, $\delta S N R 2$, and $\delta L 4$ series. As the representative value from analysis of $\delta L 4$ series will be used the mean of $h_{L 1}$ and $h_{L 2}$ (9) obtained from two dominant spectral peaks in $\delta L 4$ spectra. The reference height $h_{G}$ applied for snow depth sensing in (8) was estimated as mean value from of effective antenna height determined in time span without snow cover. It is worth to mention that due to terrain topography the estimated $h_{G}$ from observations in selected azimuth sector could be significantly different from the value evaluated from heights of pillar, antenna reference point and antenna phase centre given in Table 1.

The analysed period for GANP permanent station covers the interval from Dec. 1, 2011 till March 3, 2012. Snow depth manual measurements are adopted from meteo-service at Poprad-Tatry international airport (www.weatheronline.co.uk), $6.5 \mathrm{~km}$ away from GANP site. Since the elevation of Poprad-Tatry airport $(696 \mathrm{~m})$ is similar to Gánovce elevation and both sites are situated in the same climatic zone, we expect that the snow depth data are representative also for GANP GNSS permanent station. Descending satellite PRN 23 (elevation angle $\theta<30^{\circ}$ ) was continuously observed each day in azimuth sector from $80^{\circ}$ to $110^{\circ}$ during the whole analysed interval. Reference height $h_{G}=2.90 \mathrm{~m}$ for this azimuth sector was estimated from 15 days without snow cover.

The effective heights $h_{S 1}, h_{S 2}$ and $h_{L 4}$ above the reflecting surface were estimated independently for each day separately from $\delta S N R 1, \delta S N R 2$, and $\delta L 4$ series. Amplitudes of dominant frequencies for $\delta S N R 1$ series are in range $10-20$ (in linear scale), amplitudes of dominant frequencies for $\delta S N R 2$ are in range $2-4$ (in linear scale). Amplitudes for two dominant peaks of $\delta L 4$ are from 3 to $8 \mathrm{~mm}$.

In majority of $\delta S N R 1$ and $\delta S N R 2$ series only one peak was dominating. Amplitudes related to other non-dominating peaks are less than $70 \%$ of the amplitude of the main peak. In such case the $h_{S 1}, h_{S 2}$ were computed from the frequencies relater to major peak in each series. For several $\delta S N R 1$ or $\delta S N R 2$ series the dominant peak leads to unrealistic estimate of $h$ or more peaks with similar amplitudes are visible in the spectra (about 5 such non-standard situations occurred in the analyzed interval of PRN23 series). Such cases were solved individually (setting the polynomial of higher order up to $n=10$ for removing the long-term effects or by shortening the interval up to $50 \%$ of the original length to remove the anomalous part of the series).

Analysis of the $\delta L 4$ series is more complex. Firstly, the amplitudes of two dominant peaks are approximately equal and affiliation to $\omega_{L 1}$ or $\omega_{L 2}$ should take into consideration the expected effective antenna height above the reflecting surface. Secondly, the occurrence of more than two dominant peaks with approximately equal amplitudes was observed in about $5 \%$ of the analyzed $\delta L 4$ series. We assume that it is consequence of not completely removing ionosphere effect by polynomial approximation. Such situations were solved individually, usually by shortening the analyzed $\delta L 4$ series or by selection of second and third dominant peaks for evaluation of $h_{L I}$ and $h_{L 2}$.

Heights above the reflecting surface $h_{S 1}, h_{S 2}$ and $h_{L 4}$ estimated from analyses of PRN23 observations at GANP permanent station are plotted in Figure 8. The progressive reduction of effective antenna heights $h$ after January 10, 2012 is clearly visible as well as the progressive increase of $h$ after February 20,2012. The biases among antenna heights derived from three different observables - SNR1, SNR2 and L4 are worth noticing. The differences between the pairs of series are not stable and also some outliers can be identified.

The snow depth inferred from GPS multipath analysis and the manual snow depth measurements are shown in Figure 9. The GPS data for every day are evaluated as mean values and their standard errors from combination of $h_{S N O W}$ obtained from SNR1, SNR2 and L4 analyses. The multipath analysis yields series consistent with manual snow depth measurements, the $\sim 10 \mathrm{~cm}$ systematic difference between the two types of data is observed from February 1 till 20, 2012. Such difference can be caused by the fact that the localities of manual and GPS-inferred snow depths are separated about $6.5 \mathrm{~km}$.

The analyzed GPS data for LIE1 permanent station are from interval Nov. 28, 2012 till Feb. 27, 2013. However, due to technical problems gaps for 1 or 2 days occurred and the snow dept from GPS was not evaluated. Snow depth manual measurements 
performed daily, $30 \mathrm{~m}$ away from GPS antenna monumentation are provided by Slovak Hydrometeorological Institute.

Rising satellite PRN 15 (elevation angle $\theta<30^{\circ}$ ) was continuously observed in azimuth sector from $295^{\circ}$ to $305^{\circ}$. Reference height $h_{G}=3.55 \mathrm{~m}$ for this azimuth sector was estimated from 20 days without snow cover (from Dec. 18, 2012 to Jan. 6, 2013). The estimated effective antenna heights $h_{S 1}, h_{S 2}$ and $h_{L 4}$ representing the outputs of spectral analyses of series SNR1, SNR2 and L4 at the GPS site LIE1 are plotted in Figure 10. Consistency between $h_{S 1}$ and $h_{S 2}$ in the period without snow is better (RMS difference is 0.02 $\mathrm{m})$ than in periods with snow cover (RMS difference is $0.05 \mathrm{~m}$ ). For periods with snow cover (characterized by $h_{S 1}<h_{G}$ and $h_{S 2}<h_{G}$ ) the non constant biases between $h_{S 1}$ and $h_{S 2}$ reaching $\pm 0.10 \mathrm{~m}$ are observed. The $h_{L 4}$ values are more scattered, however generally they follow the pattern represented by $h_{S 1}$ and $h_{S 2}$. Figure 11 shows the comparison of manual snow measurements and the values $h_{S N O W}$ from the multipath analysis obtained as mean of SNR1, SNR2 and L4 inferred estimates. The GPS data mimic the manual measured snow heights and they are sensitive to sudden snow depth increase and snow melting. Biases between the two series are remarkable after Feb. 8, when the GPS multipath indicates faster snow melting than the manual measurements. However, the possible effect of local topography and larger area representing the multipath data when compared to the manual snow depth sensing may be responsible for such discrepancies.

\section{CONCLUSIONS}

Information about the actual snow depths is valuable for studying hydrological and climate cycles and for completing the meteorological data related to the long-term monitored GNSS site. The utilization of GPS multipath for snow depth sensing in the vicinity of GNSS permanent station gives a possibility to obtain new type of information without necessity to perform additional observations and do not require additional costs for equipment. The information about snow depth is important also for geodynamic investigations for reduction or interpretations of observations using absolute or relative gravimeters. We have shown that in areas with non-ideal environment for multipath analyses (sites surrounded by buildings, structures and un-even topography) can be obtained relevant snow depth series representing with sufficient reliability the actual situation. Our approach is based on analysis of three independent observation series of setting or ascending satellite observed in narrow azimuthal sector with no significant obstacles, namely the signal-to-noise ratio related to L1 and L2 frequencies (SNR1 and SNR2) and the geometry-free carrier phase linear combination L4. Each series is analyzed separately and the representative value for snow depth for each day is evaluated as mean of three independently estimated effective antenna heights. Mean errors of snow depth estimated from GPS multipath analyses are from 0.02 to $0.12 \mathrm{~m}$. The consistency of GPS estimated snow depth and manual snow cover measurements we found in the range of mean errors of GPS $h_{\text {SNOW. However we noticed several biases }}$ between the GPS and manually measured snow depths reaching $0.10 \mathrm{~m}$ and repeating for several succeeding days. We believe that analyses of multipath performance at GNSS permanent stations and their application for study of environment in the vicinity of antenna monumentation will contribute to better interpretation of observed changes related to geodynamics of monitored sites.

\section{ACKNOWLEDGEMENT}

This work was supported by the grant No. 1/0642/13 of the Grant Agency of Slovak Republic VEGA.

\section{REFERENCES}

Billich, A., Larson, K.M. and Axelrad, P.: 2008, Modelling GPS phase multipath with SNR: Case study from the Salar de Uynni, Bolivia. Journal of Geophysical Research, 3 B0440, DOI: 10.1029/2007/JB005194.

Estey, L. and Meertens, C. M.: 1999, TEQC: The multipurpose toolkit for GPS/GLONASS data. GPS Solutions 3 (1), 42-49. DOI: 10.1007/PL00012778

Gurtner, W.: 1994, RINEX: The Receiver-Independent Exchange Format. GPS World, 5, Number 7.

Hofmann-Wellenhof, B., Lichtenneger, H. and Collins, J.: 2001, GPS - Theory and Practice, $5^{\text {th }}$ edition. Springer, New York, 382 pp.

Larson, K.M., Small, E., Gutmann, E., Billich, A., Axelrad, P. and Braun, J.: 2008, Using GPS multipath to measure soil moisture fluctuations: initial results. GPS Solutions, 12, 173-177. DOI: 10.1007/s10291-007-0076-6

Larson, K.M., Gutmann, E.D., Zavorotny, V.U., Braun, J.J., Williams, M.W. and Nievinski, F.G.: 2009, Can we measure snow depth with GPS receivers? Geophysical Research Letters, 36, L17502. DOI: 10.1029/2009GL039430.

Larson, K.M. and Nievinski, F.G.: 2012, GPS snow sensing: results from the EarthScope Plate Boundary Observatory. GPS Solutions, 16. DOI: $10.1007 /$ s10291-012-0259-7

Misra, P. and Enge, P.: 2001, Global Positioning System, Signals, measurements, and Performance. Gangajammuna Press, Lincoln, Massachusetts. 391 pp.

Ozeki, M. and Heki, K.: 2011, GPS snow depth meter with geometry-free linear combinations of carrier phases., Journal of Geodesy. DOI: $10.1007 / \mathrm{s} 00190-011-0511-\mathrm{x}$

Wells, D.E., Vanicek, P. and Pagiatakis, S.: 1985, Least squares spectral analysis revisited. Technical report, No. 84, Geodesy and Geomatics Engineering, University of New Brunswick, Fredericton, Canada, $35 \mathrm{pp}$. 

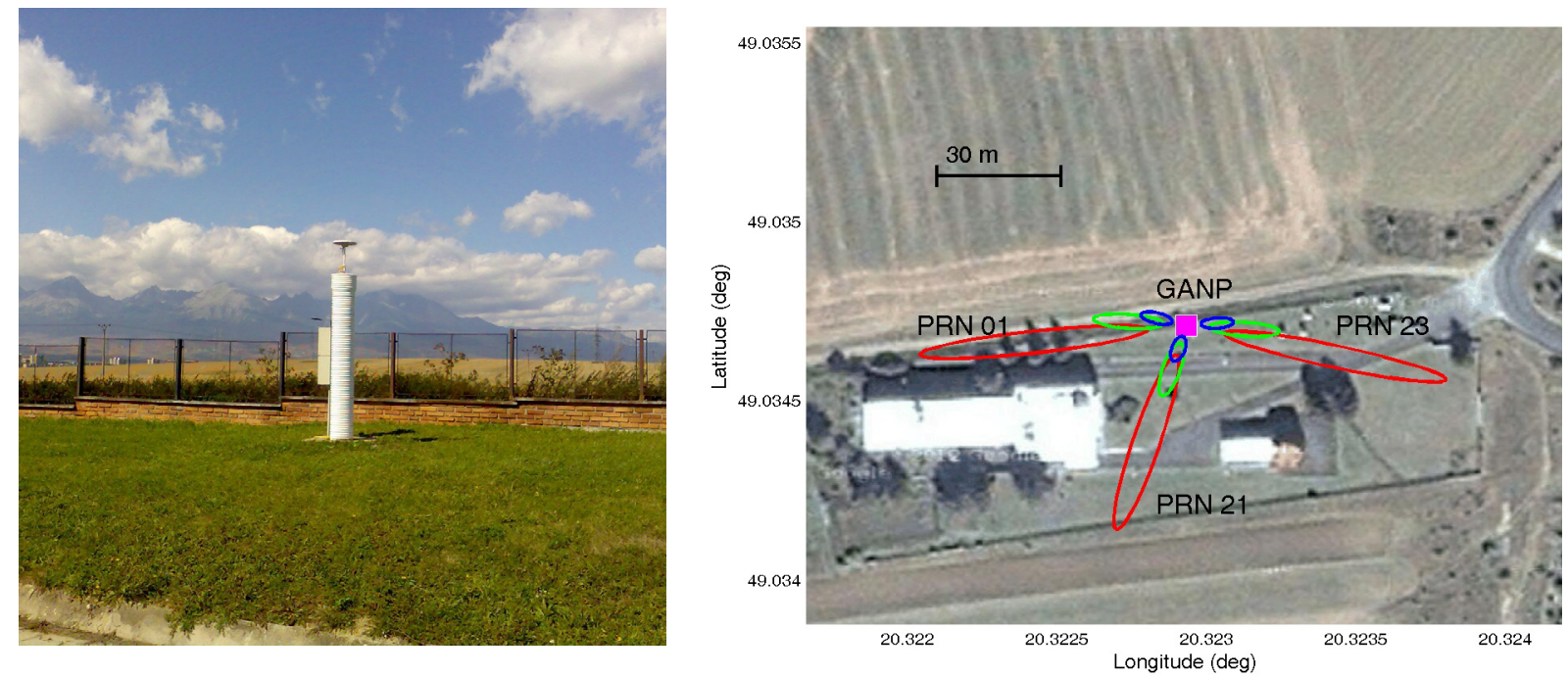

Fig. 6 Permanent GNSS station GANP and its environment. First Fresnel zones for $\theta=7^{\circ}$ (red), $\theta=10^{\circ}$ (green) and $\theta=25^{\circ}$ (blue) are shown for observed satellites and azimuth sectors with reliable multipath reflections suitable for further analyses.
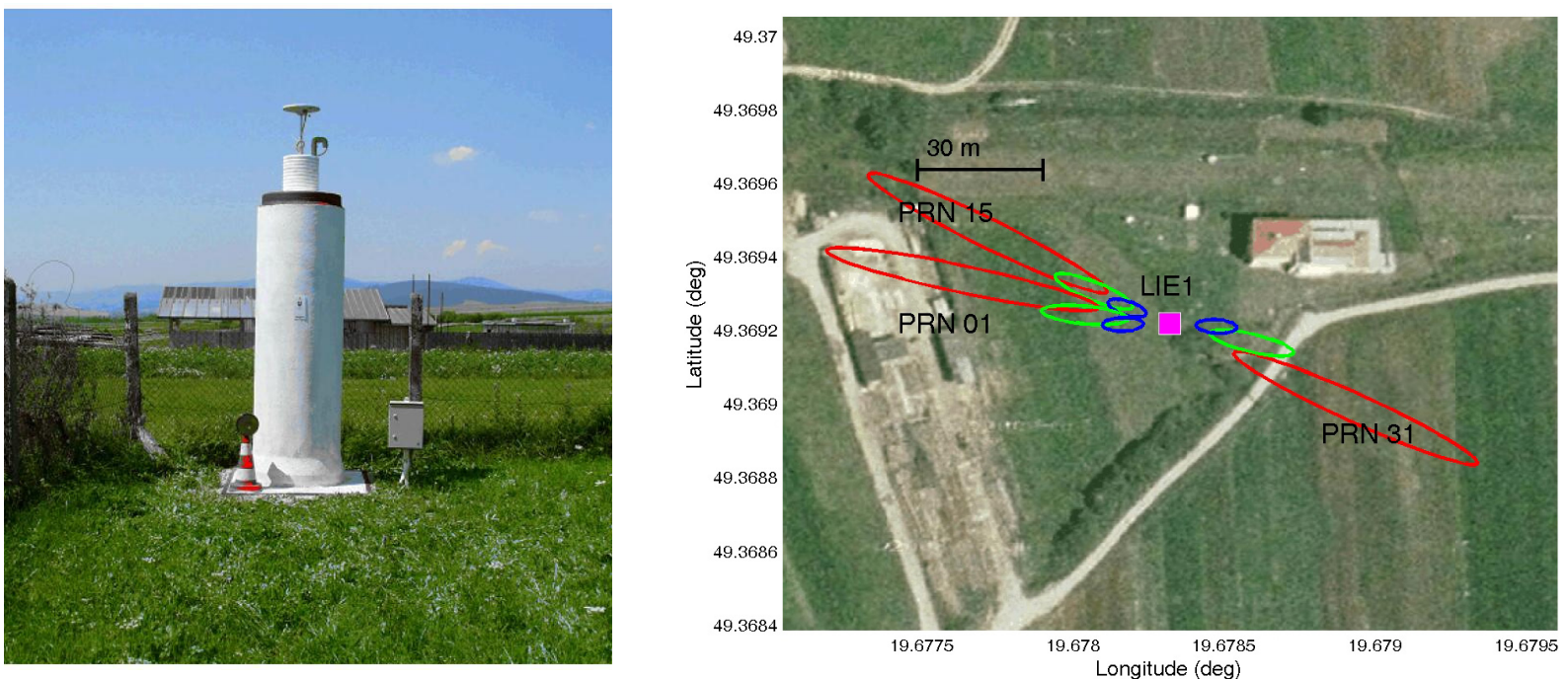

Fig. 7 Permanent GNSS station LIE1 and its environment. First Fresnel zones for $\theta=7^{\circ}$ (red), $\theta=10^{\circ}$ (green) and $\theta=25^{\circ}$ (blue) are shown for observed satellites and azimuth sectors with reliable multipath reflections suitable for further analyses. 


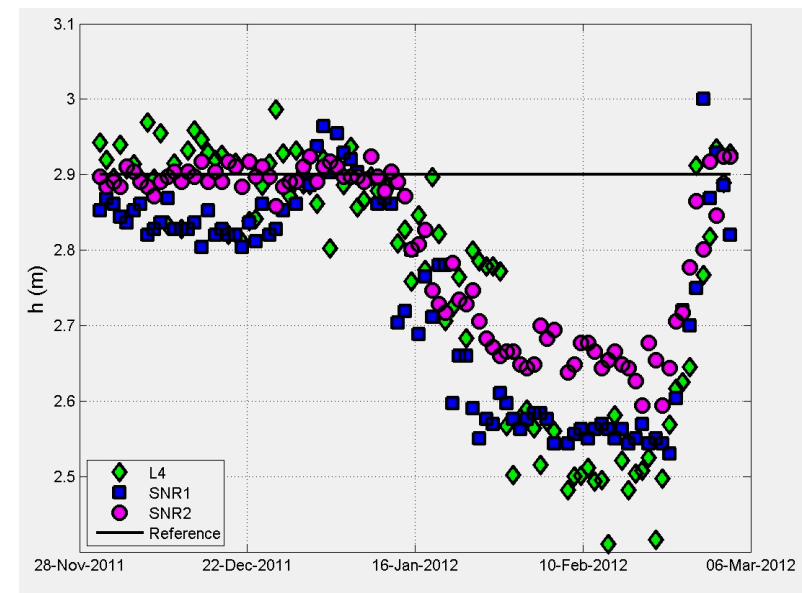

Fig. 8 Effective antenna heights above the horizontal reflection surface at permanent station GANP estimated form multipath analyses of $\delta S N R 1, \delta S N R 2$ and $\delta L 4$ series of PRN23 during the winter season 2011/2012.

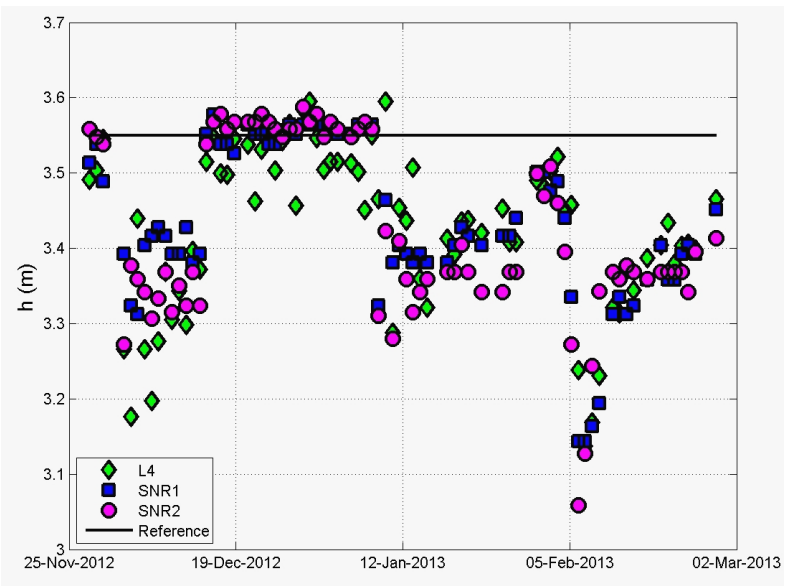

Fig. 10 Effective antenna heights above the horizontal reflection surface at permanent station LIE1 estimated form multipath analyses of $\delta S N R 1, \delta S N R 2$ and $\delta L 4$ series of PRN15 during the winter season 2012/2013.

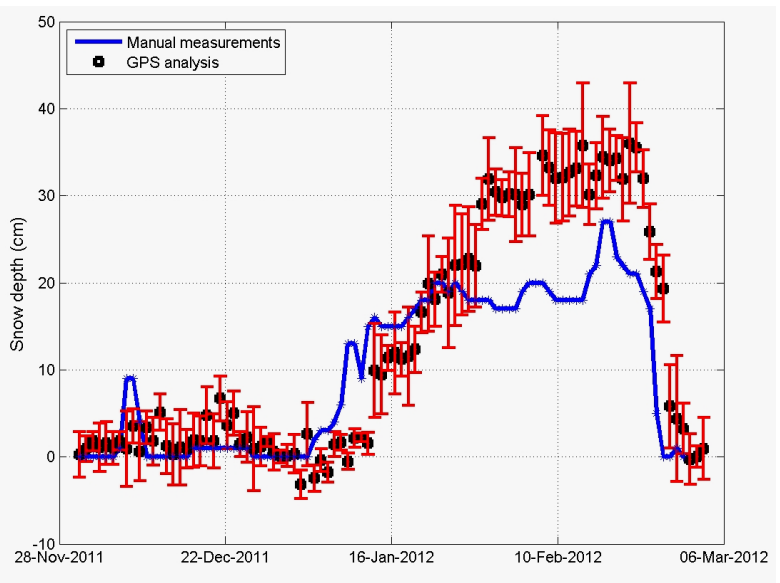

Fig. 9 Snow depths and their mean errors obtained form multipath GPS analyses of PRN23 at permanent station GANP and manual snow depth measurements at Poprad-Tatry airport.

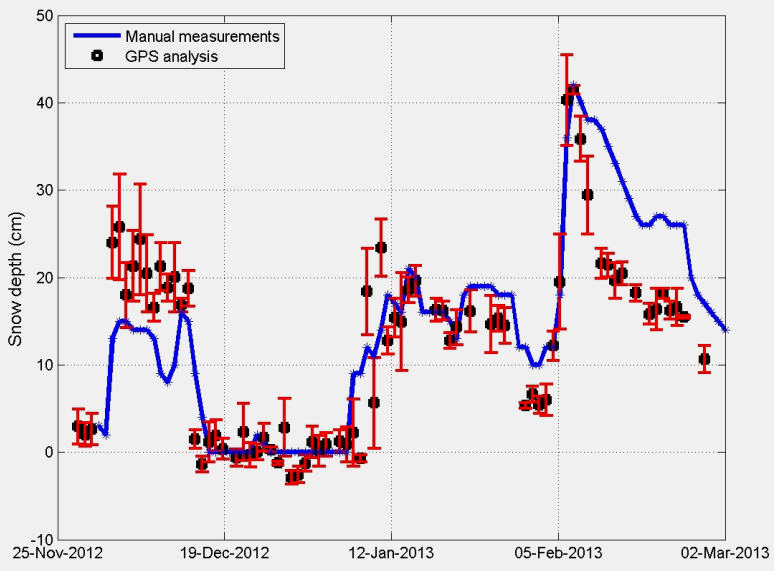

Fig. 11 Snow depths and their mean errors obtained form multipath GPS analyses of PRN15 at permanent station LIE1 and manual snow depth measurements. 